\section{Perioperative analgesic management of thoracic surgery in dogs: A descriptive study of 71 cases (2015-2018)}

\section{Luis Correa, Felipe Marquez, Federico Corletto}

Dick White Referrals, Six Mile Bottom, United Kingdom

\section{OBJECTIVES}

The aim of this study was to report the analgesic management and post-operative analgesia requirements of a group of dogs that underwent thoracic surgery.

\section{METHODS}

Clinical records of dogs that had sternotomy or intercostal thoracotomy (2015-2018) were retrospectively reviewed looking at perioperative analgesia.

\section{RESULTS}

Seventy-one dogs met inclusion criteria, 33 undergoing intercostal thoracotomy and 38 undergoing sternotomy. Locoregional anaesthesia was used in 69 cases: peripheral nerve blocks ( $n=61$ ), block in association with epidural $(n=2)$, epidural alone $(n=2)$ and epidural catheters $(n=4)$. Intercostal (32.4\%) and parasternal (25.4\%) nerve blocks were most commonly performed. Other included: infiltrative $(14.1 \%)$, thoracic paravertebral $(14.1 \%)$, serratus plane (9.8\%) and pectoralis (4.2\%). In 48 surgeries, including the two cases without locoregional analgesia, continuous rate infusions of opioids ( $n=30$ ), dexmedetomidine ( $n=8$ ), ketamine $(n=7)$ and lidocaine $(n=3)$ were used. Of those, $72.9 \%$ were started pre-emptively. The overall complication rate was $52.1 \%$, with intra-operative hypotension most commonly found $(n=24)$. The median pain score and median highest pain score on the first $24 \mathrm{~h}$ postoperatively were $2.47(1.1-5.3)$ and $3.5(2-8)$, respectively. Methadone was given based on pain checks in $36.6 \%$ of cases median dose $0.19 \mathrm{mg} / \mathrm{kg}$ and $0.95 \mathrm{mg} / \mathrm{kg} / 24 \mathrm{~h}$. Eight dogs required rescue analgesia, in a total of ten rescue interventions. Fifty-three dogs had postoperative NSAIDs, while four cases had steroids and 14 had no anti-inflammatory administered. The perioperative mortality rate was $5.6 \%$.

\section{STATEMENT (CONCLUSIONS)}

This research outlines the perioperative analgesia management of thoracic surgery in dogs, providing recent clinical context for this procedure and setting a starting point for further studies.

\section{Assessment of intra-} operative nociception in dogs. An observational pilot study

\section{Elliot Pye ${ }^{1}$, Matt Gurney ${ }^{2}$}

1 University of Nottingham, Nottingham, United Kingdom

2 Anderson Moores Veterinary Specialists, Winchester, United Kingdom

\section{OBJECTIVES}

Assessment of nociception under anaesthesia is essential to guide interventional analgesic administration, but guidelines for when to intervene are anecdotal. This pilot study aimed to demonstrate changes in basic physiological parameters used to assess nociception in dogs during anaesthesia and response to interventional analgesia.

\section{METHODS}

Baseline heart rate, respiratory rate $\&$ arterial blood pressure (direct, oscillometric or Doppler) and end-tidal carbon dioxide concentration were recorded in 35 dogs prior to surgical incision. Following incision if values exceeded $20 \%$ of baseline, suggesting nociception, further analgesia was provided. The analgesic intervention was at the discretion of the anaesthetist and not dictated by the study.

\section{RESULTS}

Changes indicative of nociception occurred in 54\% $(19 / 35)$. Of these, $31 \%(6 / 19)$ reacted on more than one occasion. Heart rate increased first in $51 \%$ of cases by a mean of $66 \%$ (20-136\%). Respiratory rate increased first in $42 \%$ of cases by a mean of $77 \%$ (20-190\%). Where direct blood pressure was measured, this increased concurrently with heart rate. Oscillometric blood pressure was a late indicator of nociception. There were 35 episodes requiring intervention and analgesics were deemed effective in $94 \%$ of episodes (33/35), although the primary parameter only returned to within $10 \%$ of baseline in 17\% (6/35). The most common adverse effect of interventional analgesics was short-duration apnoea.

\section{STATEMENT (CONCLUSIONS)}

Heart rate was the earliest indicator of nociception but the greatest change was seen in respiratory rate. Further work is required assessing which interventional analgesic is most effective and has the fewest adverse effects. 